August 2006

\title{
A phase II study of acute toxicity for Celebrex(TM) (celecoxib) and chemoradiation in patients with locally advanced cervical cancer: Primary endpoint analysis of RTOG 0128
}

\author{
David K. Gaffney \\ University of Utah \\ Kathryn Winter \\ Radiation Therapy Oncology Group \\ Adam P. Dicker \\ Thomas Jefferson University \\ BrigditeethAildeld additional works at: https://jdc.jefferson.edu/radoncfp \\ '^’רe Forest University \\ Part of the Radiology Commons \\ Partciags \$fiflow how access to this document benefits you \\ University of Texas
}

\section{Recommended Citation}

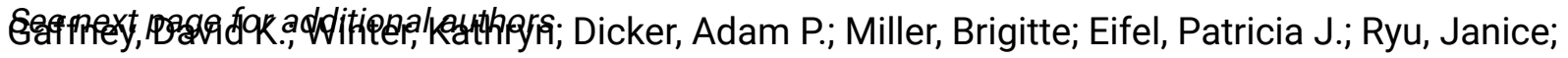

Avizonis, Vilija; Fromm, Mitch; and Greven, Kathryn, "A phase II study of acute toxicity for

Celebrex(TM) (celecoxib) and chemoradiation in patients with locally advanced cervical cancer: Primary endpoint analysis of RTOG 0128" (2006). Department of Radiation Oncology Faculty

Papers. Paper 2.

https://jdc.jefferson.edu/radoncfp/2

This Article is brought to you for free and open access by the Jefferson Digital Commons. The Jefferson Digital Commons is a service of Thomas Jefferson University's Center for Teaching and Learning (CTL). The Commons is a showcase for Jefferson books and journals, peer-reviewed scholarly publications, unique historical collections from the University archives, and teaching tools. The Jefferson Digital Commons allows researchers and interested readers anywhere in the world to learn about and keep up to date with Jefferson scholarship. This article has been accepted for inclusion in Department of Radiation Oncology Faculty Papers by an authorized administrator of the Jefferson Digital Commons. For more information, please contact: JeffersonDigitalCommons@jefferson.edu. 


\section{Authors}

David K. Gaffney, Kathryn Winter, Adam P. Dicker, Brigitte Miller, Patricia J. Eifel, Janice Ryu, Vilija Avizonis, Mitch Fromm, and Kathryn Greven 


\title{
A PHASE II STUDY OF ACUTE TOXICITY FOR CELEBREXTM (CELECOXIB)AND CHEMORADIATION IN PATIENTS WITH LOCALLY ADVANCED CERVICAL CANCER: PRIMARY ENDPOINT ANALYSIS OF RTOG 0128
}

\author{
DAVID K. GAFFNEY, M.D., PH.D., ${ }^{*}$ KATHRYN WINTER, M.S., $\dagger$ ADAM P. \\ DICKER, M.D., PH.D.,† BRIGITTE MILLER, M.D.,§ PATRICIA J. EIFEL, M.D.," \\ JANICE RYU, M.D.,II VILIJA AVIZONIS, M.D.,\# MITCH FROMM, M.D., ${ }^{* *}$ AND \\ KATHRYN GREVEN, M.D. $\dagger \dagger$ \\ *Department of Radiation Oncology, Huntsman Cancer Hospital, University of Utah, Salt Lake City, UT; \\ $\dagger$ Statistical Department, Radiation Therapy Oncology Group, Philadelphia, PA; $\ddagger$ Bodine Center for Cancer \\ Treatment, Thomas Jefferson University Hospital, Philadelphia, PA; §Department of Obstetrics and \\ Gynecology, Wake Forest University School of Medicine, Winston-Salem, NC; "Department of Radiation \\ Oncology, The University of Texas, M.D. Anderson Cancer Center, Houston, TX; IDepartment of \\ Radiation Oncology, University of CA Davis Cancer Center, Davis, CA; \#Department of Radiation \\ Oncology, LDS Hospital Radiation Center, Salt Lake City, UT; **Akron General Medical Center, Akron \\ Radiation Oncology Associates Inc., Akron OH; ††Department of Radiation Oncology, Wake Forest \\ University School of Medicine, Winston-Salem, NC
}

\begin{abstract}
Purpose: To determine treatment-related acute toxicity rates in patients with locally advanced cervical cancer treated by oral celecoxib, i.v. cisplatin and 5-FU, and concurrent pelvic radiation therapy.

Methods and Materials: Eligible patients on this RTOG Phase I-II study for advanced cervix cancer included FIGO Stage IIB-IVA or patients with FIGO Stage IB through IIA with biopsy proven pelvic node metastases ortumor size $>5 \mathrm{~cm}$. Patients were treated with pelvic radiotherapy and brachytherapy. Celecoxib was prescribed at $400 \mathrm{mg}$ twice daily beginning on day 1 for 1 year. Cisplatin (75 $\mathrm{mg} / \mathrm{m} 2)$ and $5-\mathrm{FU}$ ( $1 \mathrm{~g} / \mathrm{m} 2$ for 4 days) were administered every 3 weeks times 3 . The primary end point of the study was treatment related toxicity.

Results: Between August 2001 and March 2004, 84 patients were accrued to the study and 77 patients were evaluable for toxicity. Regarding the primary end point, toxicities were observed in the following areas: blood/bone marrow (16), gastrointestinal (14), pain (7), renal/genitourinary (6), cardiovascular (3), hemorrhage (1), and neurologic (1). For the first 75 evaluable patients, a toxicity failure was identified in 36 patients for a rate of $48 \%$.

Conclusions: Celecoxib at $\mathbf{4 0 0} \mathrm{mg}$ twice daily together with concurrent cisplatin and 5-FU and pelvic radiotherapy has a high incidence of acute toxicities. The most frequent toxicities were hematologic. Albeit, the toxicity was deemed excessive in this trial, the rate of toxicities was not too different compared to other recent experiences with concurrent chemoradiation for advanced cervix cancer.
\end{abstract}

Keywords: Cervix, Radiation therapy, Celecoxib, Acute toxicity. 


\section{INTRODUCTION}

Chemoradiation has been shown to improve overall survival in women with advanced cervix cancer (1-7). Despite a dramatic improvement in overall survival, approximately one-third of patients with advanced cervix cancer will have failed therapy within 2 years (3). Thus, improvement in the treatment of advanced cervix cancer is desperately needed. In the United States, approximately 10,370 new cases of cervical cancer will be diagnosed and of these, greater than 3,710 will die from the disease (8). Worldwide, cervix cancer continues to be the second most frequent cause of cancer and cancer-related mortality in women. Only breast cancer has a higher incidence and mortality (9). Thus, improvements in survival rates of women with cervix cancer may translate into a significant impact on women's health worldwide. Targeted therapies including Cyclooxygenase-2 (COX-2) inhibition may be promising approaches to achieve this end.

COX-2 is cytokine inducible whereas cyclooxygenase- 1 is constitutively expressed. Enhanced COX-2 expression has a key role in the development of edema by impeding blood flow and causing immunomodulation that is observed in pathologically altered disease states (10-11). COX-2 is over expressed in a wide variety of different tumors including cervix carcinomas and is associated with a poor outcome (12-15). Inhibition of COX-2 has been found to diminish tumor growth in a myriad of ways including promoting apoptosis, inhibiting vascular endothelial growth factor (VEGF), inhibiting new vessel growth, and sensitizing cells to radiation (16-24). Animal models have shown that COX-2 inhibition will improve the response to radiotherapy without markedly affecting normal tissue radiation response (25-26). Radiation of cells in vitro have also shown to increase COX-2 expression and its enzymatic product, prostaglandin E2 (PGE2) (27). Additionally, a Phase III randomized trial in familial adenomatous polyposis patients showed that 6 months of Celecoxib versus placebo significantly diminished the size and number of polyps (28). Thus, we proceeded to perform a trial in patients with advanced cervical carcinoma using i.v. cisplatin and 5-fluorouracil (5-FU) chemotherapy with concurrent celecoxib. The primary goal was to determine treatment related toxicity.

\section{METHODS AND MATERIALS}

\section{Patient eligibility}

Patients were considered eligible who had histologic proof of squamous cell carcinoma, adenocarcinoma, or adenosquamous carcinoma of the uterine cervix, International Federation of Gynecology and Obstetrics (FIGO) Stage IIB through IVA disease or patients with FIGO Stage IB to IIA who have biopsy proven pelvic lymph node metastasis or tumor size $\geq 5 \mathrm{~cm}$. Patients were required to have a Zubrod performance status of 0 to 2 and no disease outside of the pelvis. Laboratory values must be as follows: White blood cell count greater than or equal to $3000 / \mathrm{mm}^{3}$, absolute neutrophil count greater than $1500 \mathrm{~mm}^{3}$, platelets greater than $100,000 \mathrm{~mm}^{3}$, total bilirubin less than $1.5 \mathrm{mg} / \mathrm{dl}$, serum creatinine less than $1.5 \mathrm{mg} / \mathrm{dl}$, aspartate aminotransferase or alanine aminotransferase less than or equal to $2.5 \times$ the upper normal limit, serum calcium less than $1.3 \times$ the institutional upper normal limit, creatinine clearance greater than or equal to $50 \mathrm{cc} / \mathrm{min}$. All patients were required to sign a study-specific informed consent. 
Patients were considered ineligible for the protocol if any of the following applied: Prior or simultaneous malignancy unless disease free greater than 3 years, medical illness preventing the use of full dose chemotherapy, carcinoma of the cervix with histology showing small cell, carcinoid cell, clear cell, adenoid cystic carcinoma, previous medical or psychiatric illness, which would prevent informed consent, patients known to be infected with human immunodeficiency virus, prior surgery for carcinoma of the cervix other than biopsy, patients with para-aortic disease, previous radiation or systemic therapy, previous hypersensitivity to celecoxibs, patients who have recently been on any COX-2 inhibitor within 2 months, taking Dilantin or lithium, active cardiac disease, patients with active gastrointestinal ulcers, inflammatory bowel disease, and pregnant or lactating females. Additionally, patients were required to have a chest X-ray within 6 weeks of entry, and a CT or MRI of the pelvis at least to the level of the renal vessels with contrast within 6 weeks before study. Cystoscopy and sigmoidoscopy were suggested for bulky lesions and pregnancy tests were required for premenopausal females.

\section{Radiation therapy}

Radiation therapy was 45 Gy to the whole pelvis in 5 weeks in 25 fractions. A four-field technique was recommended particularly when the beam energy was less than $15 \mathrm{MV}$. The involved lateral parametrium or pelvic lymph nodes were recommended to receive a boost to achieve a total dose of $60 \mathrm{~Gy}$. Techniques to limit dose to small bowel were suggested including prone positioning and a full bladder. The superior border of the pelvic field was L4/5 and the inferior border was a transverse line below the obturator foramen. The lateral border was $2 \mathrm{~cm}$ lateral to the widest true pelvic diameter. On the lateral portal, the anterior border was placed anterior to the symphysis pubis and at least 1 $\mathrm{cm}$ anterior to the common iliac nodes at L4-5, and for the posterior border the entire sacrum was recommended or at a minimum a $3 \mathrm{~cm}$ margin posterior to the greatest extent of disease.

Low dose rate (LDR) or high dose rate (HDR) brachytherapy was permitted. For LDR, 2 insertions were recommended delivering a total RT dose of $85 \mathrm{~Gy}$ to point $\mathrm{A}$, and the interval between the 2 insertions was to be 1 to 3 weeks. The third cycle of chemotherapy was recommended to be delivered with the second brachytherapy insertion if LDR was utilized. For patients receiving HDR brachytherapy, 5 fractions of 6 Gy each to point A were recommended. One fraction a week was recommended as early as Week 3 . Two insertions per week were allowed given the fractions were separated by $72 \mathrm{~h}$. Tandem and ovoids or a tandem and ring were recommended for HDR brachytherapy. Interstitial brachytherapy was allowed to treat distal vaginal disease that cannot be covered with intracavitary techniques. Multiple points consistent with ICRU 38 were evaluated for brachytherapy dosimetry including point $\mathrm{A}$, point $\mathrm{B}$, bladder, rectum, and vaginal surface. Treatment was to be completed within 56 days. The Radiologic Physics Center in Houston, Texas evaluated all implants and provided quality assurance to all centers.

\section{Chemotherapy}

Patients received cisplatin chemotherapy after i.v. hydration at $75 \mathrm{mg} / \mathrm{m}^{2}$ with a maximum dose of $150 \mathrm{mg}$ on Days 1, 22, and 43 of RT delivery. 5FU was administered 
at $1 \mathrm{gm} / \mathrm{m}^{2}$ for 4 days either by bolus infusion or continuous infusion on Days 2-5, Days 23-26, and Days 44-47. Hematopoietic growth factors were permitted, but not specifically endorsed. Celebrex ${ }^{\mathrm{TM}}$ was to start on Day 1 of radiotherapy (RT), and continue daily for 12 months (400 mg p.o. b.i.d., total $800 \mathrm{mg}$ daily). The AM dose was to be given $3 \mathrm{~h}$ before RT. If LDR brachytherapy was utilized, the third cycle of chemotherapy was to be delivered at the time of the implant.

\section{Statistics}

The primary endpoint of treatment-related toxicity for this trial included the following: (1) Grade 3 nausea and vomiting or diarrhea despite medical intervention; (2) Grade 4 neutropenia or leukopenia persisting for greater than 7 days; (3) Grade 3 anemia or thrombocytopenia; and (4) Grade 3 gastrointestinal (GI), renal, cardiac, pulmonary, hepatic and neurologic toxicity. Chemotherapy and acute RT toxicities were scored according to the Common Toxicity Criteria version 2.0 (CTC v. 2.0) criteria. Late RT toxicities were scored according to the Radiation Therapy Oncology Group/European Organisation for Research and Treatment of Cancer (RTOG/EORTC) Late Morbidity Scoring Schema. Based on the chemoradiation arm from RTOG 90-01, about 22\% of patients in the chemoradiation arm experienced the 4 toxicities above. Assuming $20 \%$ of the toxicity is considered tolerable, we consider a toxicity rate of $35 \%$ or above as excessive. Seventy-five evaluable cases provided a $5 \%$ chance of rejecting the treatment when the severe toxicity rate is $20 \%$, and $90 \%$ chance of rejecting the treatment when the severe toxicity rate is $35 \%$. Considering $10 \%$ ineligible or lack-of-data cases, the total sample size was set at 83 . Early stopping rules for excessive toxicity were in place as well as routine interim reporting every 6 months. All eligible patients starting protocol therapy will be included in the analyses. Based on Flemming's method and assuming that the study did not meet an early stopping rule, the treatment will be rejected for excessive toxicity if there are 34 or more cases with the primary endpoint specified toxicity (29).

Table 1. Patient characteristics $(n=77)$

\begin{tabular}{lrr}
\hline $\begin{array}{c}\text { Age (years) } \\
\text { Median } \\
\text { Range }\end{array}$ & \multicolumn{2}{c}{45} \\
& \multicolumn{2}{c}{$24-68$} \\
& $n$ & $\%$ \\
Zubrod & & \\
0 & 57 & 74 \\
1 & 18 & 23 \\
2 & 2 & 3 \\
FIGO Stage & & \\
IB & 18 & 23 \\
IIA & 3 & 4 \\
IIB & 40 & 52 \\
IIIB & 13 & 17 \\
IVA & 3 & 4 \\
\end{tabular}




\section{RESULTS}

Between August 2001 and March 2004, 84 patients were accrued to the study and 77 patients were evaluable for toxicity. Four patients were ineligible, 2 received no protocol therapy and 1 withdrew consent. The median follow-up for all patients for this analysis is 18 months. The median age was 45 years (Table 1). The stage distributions were: IB 23\%; IIA 4\%; IIB 52\%; IIIB 17\%, and IVA 4\%. In December of 2004, the drug company alerted RTOG that an excess risk of cardiovascular complications was observed on previous Phase III trials employing COX-2 inhibitors. All cardiovascular complications were reviewed and none were found to be likely related to celecoxib; nevertheless, due to a small number of patients (7) potentially remaining on the maintenance phase (celecoxib therapy only) of the protocol at that time it was deemed reasonable to discontinue maintenance celecoxib therapy at that time. The potential harm was felt to be greater than the potential benefit for the few remaining weeks of celecoxib therapy.

A four-field external beam technique was used in 93\%, and an AP:PA technique in 7\%. Protocol treatment compliance is shown in Table 2. Radiotherapy and brachytherapy was performed per protocol or with a minor deviation in $79 \%$ and $82 \%$ of patients, respectively. No implant was delivered in 8 of 77 patients (10\%). This was the first RTOG cervix cancer trial that permitted HDR brachytherapy and it was utilized in 35\% of patients receiving brachytherapy. The median dose to Point A for patients that received a LDR brachytherapy was $84.98 \mathrm{~Gy}$, while the median doses to bladder and rectum were $65.50 \mathrm{~Gy}$ and $66.00 \mathrm{~Gy}$, respectively. For patients that received HDR brachytherapy, the median doses to Point A, bladder and bowel were $75.00 \mathrm{~Gy}, 62.05 \mathrm{~Gy}$, and $62.39 \mathrm{~Gy}$, respectively. Biologically equivalent dose calculations were used employing the linearquadratic formula assuming an alphabeta ratio of 10 for tumors and 2 for normal tissues (30). For acute reacting tissues or tumor this yielded an equivalent biologically equivalent dose of $101.1 \mathrm{~Gy}\left(\mathrm{BED} \mathrm{Gy}{ }_{10}\right.$ ) for both HDR and LDR; however, the biologically equivalent doses for late responding tissues were greater for HDR than LDR; 205.5 BED $\mathrm{Gy}_{2}$ and 165.5 BED Gy ${ }_{2}$, respectively. The median overall RT treatment time in this trial was 44 days, ranging from 31 to 74 days, which corresponded to 41 days (31-74 days) for LDR patients and 45 days (38-70 days) for HDR patients.

Chemotherapy compliance was more variable with only $7 \%$ of patients receiving full protocol dose of all 3 cycles. Three cycles of at least $80 \%$ of the protocol specified cisplatin and 5-FU doses each were able to be administered in 57\% and $40 \%$ of patients, respectively. Three cycles of chemotherapy was delivered to $65 \%$ of patients that completed radiotherapy treatment. The median dose of cisplatin was $355 \mathrm{mg}$. Celecoxib compliance was not high, particularly in the maintenance phase after chemoradiation, about $20 \%$ reportedly discontinued due to toxicity. The protocol specified 1 year of celecoxib at $400 \mathrm{mg}$ bid ( $800 \mathrm{mg}$ daily dose) equates to $292,000 \mathrm{mg}$, and the first quartile, median, and third quartile received were 20,000,64,800, and 196,400, respectively. The doses of celecoxib were recorded in a pill diary after instructions from member institutions. 
Table 3 describes chemotherapy and acute radiotherapy toxicity, which was graded according to the CTC v. 2.0 criteria. The most frequent Grade 3 and 4 combined toxicity was hematalogic (40/77 patients), while the most frequently observed Grade 3 toxicities were GI, hematalogic, skin, and metabolic. The worst nonhematalogic toxicity were observed in $53 \%$ and $13 \%$ of patients for Grades 3 and 4, respectively.

Table 4 describes late radiotherapy toxicity, which was scored according to the RTOG/EORTC Late Morbidity Scoring Scheme, with a relatively short median follow up of 18 months. The most common form of late radiotherapy toxicity was genitourinary followed by gastrointestinal. For Grade 3 or greater late toxicities we observed 5 bladder or genitourinary toxicities, 2 GI toxicities, 1 bone pain, 1 pelvic fracture, and 1 vaginal necrosis.

In Table 5, primary end-point toxicities are reported with toxicities observed in the following areas: blood/bone marrow (16), GI (13), pain (7), renal/genitourinary (6), cardiovascular (3), hemorrhage (1), and neurologic (1). Fortyseven toxicities were observed in 36 of the first 75 patients (48\%) and in 36 of all 77 patients (47\%). Thus, exceeding the previously defined safe limit of $35 \%$.

Table 6 describes all acute GI toxicities greater or equal to Grade 3 in recent trials employing chemoradiation. In our study, we observed a greater than or equal to Grade 3 GI toxicity in $45 \%$ of patients. This is elevated above other experiences in cooperative groups, either with cisplatin or 5-FU and cisplatin-based chemotherapeutic regimens.

Table 2. Radiotherapy, brachytherapy, and chemotherapy compliance $(n=77)$

\begin{tabular}{lrrrrrrr}
\hline & \multicolumn{2}{c}{ Radiotherapy } & & \multicolumn{2}{c}{ Brachytherapy } & & \multicolumn{2}{c}{ Chemotherapy } \\
& \multicolumn{1}{c}{$\mathrm{n}$} & $\%$ & $\mathrm{n}$ & $\%$ & & $\mathrm{n}$ & $\%$ \\
\hline Per protocol & 54 & 70 & 51 & 66 & 20 & 26 \\
Variation acceptable & 7 & 9 & 12 & 16 & 40 & 52 \\
Deviation unacceptable & 11 & 14 & 5 & 6 & 2 & 3 \\
Incomplete & 5 & 6 & 9 & 12 & 15 & 19 \\
\hline
\end{tabular}

For Radiotherapy, one patient was scored as Incomplete due to progression, and 4 patients refused to continue with protocol treatment. For Brachytherapy, eight patients did not receive an implant and one was not evaluable.

Table 3. Chemotherapy and acute radiotherapy toxicity $(n=77)$

\begin{tabular}{lcccc}
\hline & \multicolumn{3}{c}{ Grade } \\
\cline { 2 - 5 } & 1 & 2 & 3 & 4 \\
\hline Allergy/immunology & 1 & 1 & 3 & 0 \\
Auditory/hearing & 0 & 6 & 2 & 0
\end{tabular}




$\begin{array}{lrrrr}\text { Blood/bone marrow } & 9 & 23 & 22 & 18 \\ \quad \text { Hemoglobin decreased } & 4 & 11 & 4 & 2 \\ \quad \text { Leukopenia } & 1 & 8 & 12 & 8 \\ \quad \text { Lymphopenia } & 0 & 2 & 0 & 0 \\ \quad \text { Neutropenia } & 0 & 0 & 3 & 7 \\ \quad \text { Packed red blood cell } & & & & \\ \quad \text { rransfusion } & 0 & 0 & 2 & 0 \\ \quad \text { Platelet count decreased } & 3 & 2 & 1 & 1 \\ \quad \text { Hematologic-Other } & 1 & 0 & 0 & 0 \\ \text { Cardiovascular (arrhythmia) } & 0 & 0 & 0 & 1 \\ \text { Cardiovascular (general) } & 6 & 1 & 2 & 0 \\ \text { Constitutional symptoms } & 20 & 14 & 6 & 0 \\ \text { Dermatology/skin } & 10 & 15 & 14 & 0 \\ \text { Endocrine } & 2 & 4 & 0 & 0 \\ \text { Gastrointestinal } & 9 & 26 & 33 & 2 \\ \text { Hemorrhage } & 16 & 2 & 2 & 0 \\ \text { Hepatic } & 11 & 10 & 0 & 0 \\ \text { Infection/febrile neutropenia } & 3 & 5 & 5 & 3 \\ \text { Metabolic/laboratory } & 22 & 3 & 10 & 3 \\ \text { Musculoskeletal } & 1 & 1 & 0 & 0 \\ \text { Neurology } & 10 & 7 & 3 & 0 \\ \text { Ocular/visual } & 2 & 1 & 0 & 0 \\ \text { Pain } & 7 & 19 & 8 & 2 \\ \text { Pulmonary } & 2 & 3 & 0 & 0 \\ \text { Renal/genitourinary } & 22 & 15 & 7 & 0 \\ \text { Sexual reproductive function } & 1 & 3 & 0 & 0 \\ \text { Worst non-hematologic } & 6 & 18 & 41 & 10 \\ & (8 \%) & (23 \%) & (53 \%) & (13 \%) \\ \text { Worst overall } & 2 & 9 & 40 & 24 \\ & (3 \%) & (12 \%) & (52 \%) & (31 \%) \\ & & & & \end{array}$

Table 4. Late RT toxicity $(n=75)$

\begin{tabular}{lrrrr}
\hline & \multicolumn{4}{c}{ Grade } \\
\cline { 2 - 5 } & 1 & 2 & 3 & 4 \\
\hline Bladder & 6 & 3 & 3 & 2 \\
Bone & 2 & 2 & 1 & 1 \\
Kidney & 2 & 0 & 0 & 0 \\
Other & 6 & 11 & 1 & 0 \\
Skin & 6 & 0 & 0 & 0 \\
Small/large Intestine & 13 & 7 & 2 & 0
\end{tabular}



Worst overall
14
$(18 \%)$
15
$(19 \%)$
7
$(9 \%)$
3
$(4 \%)$

Abbreviation: $\mathrm{RT}=$ radiotherapy.

Table 6. Comparison of $>$ Grade 3 GI toxicities

\begin{tabular}{llcc}
\hline Study & Regimen & $\begin{array}{c}\text { Acute GI } \\
\text { toxicity } \\
\text { Grade }>3\end{array}$ & $\begin{array}{c}\text { Late GI } \\
\text { toxicity } \\
\text { Grade }>3\end{array}$ \\
\hline Current & 5FU, CDDP, and & $45.4 \%$ & $2.7 \%$ \\
RTOG 9001 & Celecoxib & & \\
GOG & 85 5FU and CDDP & $8.7 \%$ & $12.6 \%$ \\
GOG & 120 5FU, CDDP, and & $18 \%$ & $\mathrm{NR}$ \\
GOG 120 & Hydroxyurea & & NR \\
GOG 123 & CDDP weekly & $12 \%$ & $\mathrm{NR}$ \\
NCIC & CDDP weekly & $14.2 \%$ & $\mathrm{NR}$ \\
GOG 165 & CDDP weekly & $12.6 \%$ & $4.8 \%$ \\
GOG 165 & Ci5FU weekly & $25 \%$ & $\mathrm{NR}$ \\
& & $19 \%$ & $\mathrm{NR}$ \\
\hline
\end{tabular}

Abbreviations: $\mathrm{CDDP}=$ cisplatin; 5FU = 5-fluorouracil; $\mathrm{GOG}=$ Gynecologic Oncology Group; GI = gastrointestinal; NCIC = National Cancer Institute of Canada; RTOG = Radiation Therapy Oncology Group

\section{DISCUSSION}

COX-2 inhibitors have been combined with chemotherapy in a number of settings.

Celecoxib has been combined with RT in a number of settings including the treatment of lung, CNS, and GI malignancies and shown to be safe (31-34). In a Phase I study performed at MD Anderson Cancer Center in unfavorable performance nonsmall lung cancer patients treated to $66 \mathrm{~Gy}$ in 33 fractions with concurrent celecoxib, the maximally tolerated dose was not reached; and $800 \mathrm{mg}$ bid of celecoxib was observed to be safe (31). Celecoxib related toxicity was observed in 3 of 47 patients in their study. The efficacy of celecoxib in this trial along with chemoradiation is scheduled to be evaluated subsequently. One pilot study showed reasonable promise in the treatment of advanced pancreatic carcinoma cancer without increased toxicity (35). Whereas other studies have shown increased toxicity without increased efficacy in the treatment of GI malignancies with chemotherapy $(36,37)$. 
The primary toxicity in our study was hematologic. Nevertheless, we experienced a significant rate of GI toxicity in this study. Although we observed a Grade 3 rate of GI toxicity in $43 \%$ (33/77) of patients, the toxicities observed were principally diarrhea, nausea, and dehydration, many of which were able to be controlled with medical intervention. We did not experience a significant number of adverse events attributable to upper GI toxicity which more readily could implicate the celecoxib therapy. In some trials, coxibs showed no more upper GI toxicity than placebo while other studies showed no difference in GI bleeding rates compared with nonsteroidal anti-inflammatory agents (38-40). In studies such as this, it is difficult to ascribe toxicities to a single agent when patients are receiving a complex regimen of external beam and intracavitary radiotherapy and chemotherapy with 5-FU and cisplatin. Although the acute toxicity in this trial was moderately high indicating the toxicity of the regimen, it was similar to the overall rate of Grade 3 or greater toxicity seen in Gynecologic Oncology Group (GOG) 165 with either pelvic RT with weekly cisplatin or with continuous infusion 5-FU. The worst overall Grade 3 and 4 toxicity observed in this trial with daily celecoxib, cisplatin and 5-FU was $52 \%$, compared with $58 \%$ and $32 \%$ observed with weekly cisplatin and continuous infusion 5-FU in GOG 165, respectively (Table 6) (41). The higher rate of toxicities reported herein and by Lanciano et al. appear to be higher than the original reports that provided the NCI alert indicating the benefit seen with the cisplatin based regimens in the radiotherapeutic management of women with advanced cervix cancer $(1-6,41)$. This may be in part related to selection bias. An interesting study by Torres from MD Anderson demonstrated increased toxicity seen in patients treated more recently at their facility compared with patients treated on randomized trials in the 1990s (42).

In this trial, our a priori safety boundary was exceeded. It is possible that this is a substantially toxic regimen, or that our population did not match our comparison group well from the chemoradiation arm of RTOG 9001. If promising efficacy is seen with this regimen and no substantial increase in late toxicities, it may be reasonable to further test celecoxib together with chemoradiation in the treatment of carcinoma of the cervix. We recommend continued accrual to trials evaluating different biologic agents along with chemoradiotherapy or novel approaches to the treatment of advanced cervix cancer.

\section{REFERENCES}

1. Morris M, Eifel PJ, Lu J, et al. Pelvic radiation with concurrent chemotherapy compared with pelvic and para-aortic radiation for high-risk cervical cancer. $N$ Engl J Med 1999;340: 1137-1143.

2. Eifel PJ, Winter K, Morris M, et al. Pelvic irradiation with concurrent chemotherapy versus pelvic and para-aortic irradiation for high-risk cervical cancer: an update of radiation therapy oncology group trial (RTOG) 90- -1 . J Clin Oncol 2004;22:872--880.

3. Rose PG, Bundy BN, Watkins EB, et al. Concurrent cisplatinbased radiotherapy and chemotherapy for locally advanced cervical cancer. $N$ Engl J Med 1999;340:1144 -1153.

4. Whitney CW, Sause W, Bundy BN, et al. Randomized comparison of fluorouracil plus cisplatin versus hydroxyurea as an adjunct to radiation therapy in stage IIBIVA carcinoma of the cervix with negative para-aortic lymph nodes: a Gynecol 
Oncol Group and Southwest Oncology Group study. J Clin Oncol 1999;17:1339 -1348.

5. Peters WA III, Liu PY, Barrett RJ, et al. Concurrent chemotherapy and pelvic radiation therapy compared with pelvic radiation therapy alone as adjuvant therapy after radical surgery in high-risk early-stage cancer of the cervix. J Clin Oncol 2000;18:1606 -1613.

6. Keys HM, Bundy BN, Stehman FB, et al. Cisplatin, radiation and adjuvant hysterectomy compared with radiation and adjuvant hysterectomy for bulky stage IB cervical carcinoma. $N$ Engl J Med 1999;340:1154 -1161.

7. Pearcy T, Brundage M, Drouin P, et al. Phase III trial comparing radical radiotherapy with and without cisplatin chemotherapy in patients with advanced squamous cell cancer of the cervix. J Clin Oncol 2002;20:966 -972.

8. Jemal A, Murray T, Ward E, et al. Cancer Statistics, 2005. CA Cancer J Clin 2005;55:10 -30.

9. Parkin DM, Bray F, Ferlay J, et al. Global Cancer Statistics, 2002. CA Cancer J Clin 2005;55:74 -108.

10. Taketo M. Cyclooxygenase-2 inhibitors in tumorigenesis (part 1). J Natl Cancer Inst 1998;90:1529 -1536.

11. Taketo M. Cyclooxygenase-2 inhibitors in tumorigenesis (part II). J Natl Cancer Inst 1998;90:1609 -1620.

12. Ryu HS, Chang KH, Yang HW, et al. High cyclooxygenase-2 expression in stage IB cervical cancer with lymph node metastasis or parametrial invasion. Gynecol Oncol 2000;76:320-325.

13. Gaffney DK, Holden JA, Davis M, et al. Elevated cyclooxygenase-2 expression correlate with diminished survival in carcinoma of the cervix treated with radiotherapy. Int J Radiat Oncol Biol Phys 2001;49:1213-1217.

14. Ferrandina G, Lauriola L, Distefano MG, et al. Increased cyclooxygenase-2 expression is associated with chemotherapy resistance and poor survival in cervical patients. J Clin Oncol 2002;20:973-981.

15. Chen HHW, Wu-Chou S, Cheng-Yang C, et al. Increased expression of nitric oxide synthase and Cycloxygenase- 2 is associated with poor survival in cervical cancer treated with radiotherapy. Int J Radiat Oncol Biol Phys 2005;63:1093-1100.

16. Tsujii M, Du Bois R. Alterations in cellular adhesion and apoptosis in epithelial cells overexpressing prostaglandin endoperoxide synthase-2. Cell 1995;83:493-501.

17. Elder D, Halton D, Hague A, et al. Induction of apoptotic cell death in human colorectal carcinoma cell lines by a cyclooxygenase-2 (COX-2)-selective nonsteroidal anti-inflammatory drug: independence from $\mathrm{COX}-2$ protein expression. Clin Cancer Res 1997;3:1679-1683.

18. Tsujii M, Kawano S, DuBois R. Cyclooxygenase-2 expression in human colon cancer cells increases metastatic potential. Proc Natl Acad Sci USA 1997;94:3336-3340.

19. Lund EL, Bastholm L, Kristjansen PE. Therapeutic synergy of TNP-470 and ionizing radiation: effects on tumor growth, vessel morphology, and angiogenesis in human glioblastoma multiforme xenografts. Clin Cancer Res 2000;6:971-978.

20. Masferrer JL, Leahy KM, Koki AT, et al. Antiangiogenic and antitumor activities of cyclooxygenase-2 inhibitors. Cancer Research 2000;60:1306 -1311. 
21. Gorski DH, Beckett MA, Jaskowiak NT, et al. Blockade of the vascular endothelial growth factor stress response increases the antitumor effects of ionizing radiation. Cancer Research 1999;59:3374 -3378.

22. Williams CS, Tsujii M, Reese J, et al. Host cyclooxygenase-2 modulates carcinoma growth. J Clin Invest 2000;105:1589-1594.

23. Chiarugi V, Magnelli L, Gallo O. Cox-2, iNOS and p53 as play-makers of tumor angiogenesis. Int J Mol Med 1998;2: 715-719.

24. Milas L, Kishi K, Hunter N, et al. Enhancement of tumor response to gammaradiation by an inhibitor of cyclooxygenase-2 enzyme. J Natl Cancer Inst 1999;91:1501-1504.

25. Gallo O. Re: Enhancement of tumor response to gammaradiation by an inhibitor of cyclooxygenase-2 enzyme. J Natl Cancer Inst 2000;92:346 -347.

26. Kishi K, Petersen S, Petersen C, et al. Preferential enhancement of tumor radioresponse by a cyclooxygenase-2 inhibitor. Cancer Res 2000;60:1326 -1331.

27. Steinauer KK, Gibbs I, Ning S, et al. Radiation induces upregulation of cyclooxygenase-2 (COX-2) protein in PC-3 cells. Int J Radiat Oncol Biol Phys 2000;48:325-328.

28. Steinbach G, Lynch PM, Phillips RK, Wallace MH, Hawk E, Gordon GB, et al. The effect of celecoxib, a cyclooxygenase- 2 inhibitor, in familial adenomatous polyposis. N Engl J Med 2000;342:1946 -1952.

29. Fleming TR. One-sample multiple testing procedure for Phase II clinical trials. Biometrics 1982;38:143-151.

30. Available at: http://www.radiotherapy.com/calculate/calc.html. Accessed on February 28, 2006.

31. Liao Z, Komaki R, Milas L, et al. A phase I clinical trial of thoracic radiotherapy and concurrent celecoxib for patients with unfavorable performance status inoperable/unresectable non-small cell lung cancer. Clin Cancer Res 2005 $1 ; 11: 3342-3348$.

32. Cerchietti LC, Bonomi MR, Navigante AH, Castro MA, Cabalar ME, Roth BM. Phase I/II study of selective cyclooxygenase-2 inhibitor celecoxib as a radiation sensitizer in patients with unresectable brain metastases. J Neurooncol 2005 71:73--81.

33. Govindan R, McLeod H, Mantravadi P. Cisplatin Fluorouracil, celecoxib, and RT in resectable esophageal cancer: preliminary results. Oncology 2004;18:18 -21.

34. Blanke CD, Mattek NC, Deloughery TG, et al. A phase I study of 5-fluorouracil, leucoverin, and celecoxib in patients with incurable colorectal cancer.

Prostagland Lipid Mediat 2005; 75:169 -172.

35. Milella M, Gelibter A, Di Cosimo S, et al. Pilot study of celecoxib and infusional 5-flouorourcil as second-line treatment for advanced pancreatic carcinoma. Cancer 2004;101:133-138.

36. Crane $\mathrm{CH}$, Mason K, Janjan NA, et al. Initial experience combinig cyclooxygenase- 2 inhibition with chemoradiation for locally advanced pancreatic cancer. Am J Clin Oncol 2003;S81-S84.

37. Becerra CR, Frenkel EP, Ashfaq R, Gaynor RB. Increased toxicity and lack of efficacy of Rofecoxib in combination with chemotherapy for treatment of metastatic colorectal cancer: A phase II study. Int J Cancer 2003;105:68 -72.

38. Pronai L, Hritz I, Molnar B, et al. COX-2-selective inhibitors (COXIBs): gastrointestinal safety. Int J Immunopathol Pharmacol 2003;16:23-30. 
39. Scheiman JM. Gastroduodenal safety of cyclooxygenase-2 inhibitors. Curr Pharm Des 2003;9:2197-z206.

40. Stock1 K, Cyprien L, Chang EY. Gastrointestinal bleeding rates among managed care patients newly started on cox-2 inhibitors or nonselective NSAIDs. J Manag Care Pharm 2005; 11:550 -558.

41. Lanciano R, Calkins A, Bundy BN, et al. A randomized comparison of weekly cisplatin or protracted venous infusion of Fluorouracil in combination with pelvic radiation in advanced cervix cancer: a gynecologic oncology group study. J Clin Oncol 2005;23:8289-8295.

42. Torres M, Jhingram A, Bodurka D, Levenback CF, Eifel PJ. Concurrent Chemoradiation in the Routine Management of Patients with Cervical Cancer: How does the Experience Compare with Treatment on a Prospective Randomized Trial? [Abstract]. 